\title{
Motor processes in mental rotation
}

\author{
Mark Wexler $^{\mathrm{a}, *}$, Stephen M. Kosslyn ${ }^{\mathrm{b}}$, Alain Berthoz ${ }^{\mathrm{a}}$ \\ ${ }^{a}$ Laboratoire de Physiologie de la Perception et de l'Action, CNRS/Collège de France, Paris, France \\ ${ }^{\mathrm{b}}$ Department of Psychology, Harvard University, Boston, MA, USA
}

Received 18 April 1997; accepted 5 May 1998

\begin{abstract}
Much indirect evidence supports the hypothesis that transformations of mental images are at least in part guided by motor processes, even in the case of images of abstract objects rather than of body parts. For example, rotation may be guided by processes that also prime one to see results of a specific motor action. We directly test the hypothesis by means of a dual-task paradigm in which subjects perform the Cooper-Shepard mental rotation task while executing an unseen motor rotation in a given direction and at a previously-learned speed. Four results support the inference that mental rotation relies on motor processes. First, motor rotation that is compatible with mental rotation results in faster times and fewer errors in the imagery task than when the two rotations are incompatible. Second, the angle through which subjects rotate their mental images, and the angle through which they rotate a joystick handle are correlated, but only if the directions of the two rotations are compatible. Third, motor rotation modifies the classical inverted $\mathrm{V}$-shaped mental rotation response time function, favoring the direction of the motor rotation; indeed, in some cases motor rotation even shifts the location of the minimum of this curve in the direction of the motor rotation. Fourth, the preceding effect is sensitive not only to the direction of the motor rotation, but also to the motor speed. A change in the speed of motor rotation can correspondingly slow down or speed up the mental rotation. (C) 1998 Elsevier Science B.V. All rights reserved
\end{abstract}

Keywords: Mental rotation; Motor processes

* Corresponding author. LPPA, Collège de France, 11 pl. Marcelin Berthelot, 75005 Paris, France. Tel.: +33 1 44271624; +33 1 44271382; e-mail: wexler@ccr.jussieu.fr 


\section{Mental image transformation and motor action}

By charting the time course of image processing, Shepard and co-workers (Shepard and Metzler, 1971; Cooper and Shepard, 1973) showed convincingly that mental imagery is amenable to scientific study. The transformation of objects in mental images, especially their rotation, but also translation and zooming, have been most actively studied (e.g. see Kosslyn, 1980; Shepard and Cooper, 1982). Two results stand out in this body of research: First, objects in images seem to move along continuous trajectories as they are transformed, and in fact more time is required to perform larger transformations (Shepard and Cooper, 1982); second, mechanisms used in visual perception also play a key role in visual mental imagery (e.g. Kosslyn, 1994). Shepard and his co-workers (for a review, see Shepard and Cooper, 1982) have emphasized that visual mechanisms are at the root of image transformations. Shepard and Judd (1976), for example, have found similarities between apparent motion during vision and mental rotation.

The transformation of mental images has been studied most intensely by means of what we will call the Shepard task. This task involves two stimuli that differ by a rotation and possibly by a reflection; the subject decides whether one version of the stimuli is a reflected version of the other. The most common strategy for accomplishing this task is rotation. Note that we have not said 'mental rotation' but only rotation, and for the following reason. Show the Shepard stimuli to an adult, and his or her typical reaction will be to physically, usually manually, rotate one of them until it visually matches (or doesn't match) the other. Kirsh and Maglio (1994), for instance, have shown that in the computer game Tetris, where properly orienting the 'stimuli' is crucial, such manual-visual rotation is the strategy of choice for experts. If the stimuli themselves cannot be turned for some reason, people will often try to turn their heads in order to align the retinal image of one stimulus with a memory trace of the other.

When the stimuli cannot be moved and one cannot move one's head, one can still perform the Shepard task. Shepard and many others have shown that in this situation people typically perform mental rotation. This process is, metaphorically, a 'rotation' because it faithfully represents a physical rotation, or can be mapped onto an on-going rotation through intermediate orientations, taking longer for larger angles, and so on. It is 'mental' because the external stimulus is not turning. Mental rotation is thus an operation on visual mental images.

In this article, we test the idea that the two rotation strategies in the Shepard task, the motor, external strategy and the mental, internal one, are linked: according to our hypothesis, mental rotation is a covert simulation of motor rotation. Instead of overtly performing a rotation, with the hand or the head, and seeing its results (thereby letting the external world do our computation), in mental rotation we plan the action but do not execute it overtly; instead of seeing the outcome (which we cannot do, because the action is not physically carried out), we simulate the perceptual result of our planned action, effectively short-circuiting the actionperception cycle. This hypothesis goes somewhat against the grain of thinking in cognitive science, which typically assigns motor processes, overt or covert, to the 
role of simple outputs from higher-level information processing. However, if our hypothesis is correct, parts of the motor system, far from being a simple output device for cognition, may actually be the engine driving important cognitive operations.

For our hypothesis to be plausible, a mechanism must exist that allows one to anticipate the visual consequences of making a movement (see Berthoz (1997) for a general discussion). There is both neurophysiological and behavioral evidence for such a mechanism. For example, Duhamel et al. (1992) discovered cells in the monkey brain that shift their receptive fields in the anticipation of saccades, stabilizing the visual world in the presence of an unstable retinal image. Even more pertinent for our purposes is the finding by Hietanen and Perrett (1996) that certain cells in area STP (which has important inputs from posterior parietal cortex) modulate their response to moving stimuli depending on whether the stimulus is a result of the monkey's own movement or the experimenter's (visually, the two conditions are very similar). Moreover, this is true both when the stimulus is a monkey paw (the monkey's real paw moved endogenously, or a fake paw manipulated by the experimenter), or an object (manipulated either by the monkey, or by the experimenter). Given that these STP cells distinguish self-produced from external movement, they must have access to a visual representation of the anticipated results of the motor act. In addition, Rieser and his colleagues (Rieser et al., 1986) have shown that when people memorize the positions of several objects and then walk blindfolded to a new location, they can quickly and accurately point to the new egocentric positions of the objects. This 'mental updating' seems to be automatic and difficult to inhibit; after memorizing an object's position and moving without vision, it is easier to give the object's new, mentally-updated position than the old, memorized one. Such mental updating requires that one anticipate the way a scene will look after one has changed position. The fact that this process occurs even when one is blindfolded is exactly as expected if such a process is also used in transforming visual mental images.

To restate our hypothesis: visuomotor anticipation is the engine that drives mental rotation. Mental rotation would thus recruit motor planning and anticipation, but not the cortical and subcortical mechanisms responsible for the execution of movement. If we are correct, mental rotation ought to share some of the motor system's neural substrate. But just as motor anticipation is available even in the absence of overt movement, mental rotation can be performed while the subject is perfectly still. Nevertheless, we should be able to find evidence of specific interaction between motor anticipation and mental image transformation. Several strands of indirect evidence support the existence of such an interaction. First, many brain-imaging studies of mental image transformation report activation of motor and visuomotor areas, in particular posterior parietal cortex and motor and premotor cortex. For example, Parsons et al. (1995) used positron emission tomography (PET) to study mental rotation of hands. They found activation of supplementary motor cortex and the superior premotor areas, as well as motor-related parietal regions. In addition, Cohen et al. (1996) used functional magnetic resonance imaging to study the original Shepard and Metzler (1971) task, and report activation in premotor area 6 (in half 
the subjects), hand somatosensory cortex, areas $7 \mathrm{a}$ and $7 \mathrm{~b}$ (all subjects), and area 8 ( $88 \%$ of the subjects); SMA was also activated in some participants. Most recently, Kosslyn et al. (1998) found activation in SMA, premotor cortex, and M1 when participants visualized rotating hands.

Second, activation of posterior parietal cortex has been found in almost all studies of mental image transformation. In studies of monkeys, posterior parietal cortex has been characterized as a visuomotor area par excellence, responsible for the integration of visual information in the planning and control of movement (see Andersen et al., 1997; for a review). In humans, lesions in this area lead to apraxia. In a recent study, Sirigu et al. (1996) found that parietal lesions in humans lead to a failure to accurately anticipate the details of motor action (such as its timing) in motor imagery tasks, an anticipation that is accurate in normal subjects and in patients with motor cortex lesions.

Third, Georgopoulos et al. (1989) found 'rotation' in monkey motor cortex of a population vector that guides hand movement. Converging evidence has been reported in humans (Georgopoulos and Massey, 1987): when one has to make a movement that deviates by a specified angle from a visually-presented stimulus, the movement onset time depends nearly linearly on the angle, which is what we would expect if, as in the monkey, the neuronal population vector in motor cortex had to first rotate to the target movement direction. The relation of these findings to the mental rotation of visual images could be doubted, were it not for another interesting observation (Pellizzer and Georgopoulos, 1993): the speeds of visuomotor rotation onset and of mental rotation of visual images, which vary widely between individuals, are correlated. Moreover, this correlation does not reflect a general 'mental speed' given that neither visuomotor rotation onset time nor visual mental rotation speed was correlated with the speed of a visuomotor memory task.

Fourth, additional evidence for a link between action and mental rotation comes from behavioral studies of the mental rotation of body parts, especially hands. In the task, participants are asked to identify a picture as being that of a left or a right hand. In general, people find it easier to mentally rotate body parts in ways that actual body parts can be easily rotated than in ways that would be awkward to perform physically (see Cooper and Shepard, 1975; Sekiyama, 1982; Parsons, 1987; Parsons, 1994). It has been shown recently that the same constraints apply to the mental manipulation of images of objects that are commonly manipulated with the hands, such as a screwdriver (C. de'Sperati and P. Viviani, pers. commun.) These findings, together with the physiological data already mentioned, are consistent with the hypothesis that the mental rotation of images of hands is identical with the process of motor imagery, the first-person imagination of movement (Jeannerod, 1997). Our hypothesis, however, is stronger, positing a connection between motor action and mental rotation of images of arbitrary objects, not just hands (whose transformations would more likely be related to the motor system).

The studies noted above provide a substantial, although indirect, link between motor processes and mental image transformation. The most direct study to date has been that of Wohlschläger and Wohlschläger (1998). Using a dual-task paradigm, they found that mental rotation performed in the same direction as a concurrent 
manual rotation (and about the same axis) is faster than manual rotation in the opposite direction as the manual rotation. When the two rotations, the manual and the mental, were performed about different axes, no interference was found. This result is direct evidence for the imagery-motor hypothesis. However, the link that Wohlschläger and Wohlschläger's result establishes between motor and mental rotation processes is not very specific, and thus leaves room for alternate explanations. For example, the additional time in the incompatible case could have been caused by a higher-level conflict in short-term spatial memory, which might stem from amodal representations for the two opposite rotation directions. Evidence of a more precise correspondence between motor and image rotations is necessary to test the hypothesis of a mental rotation-motor action link; such evidence must rule out higher-level conflict as the origin of the effect.

\section{Experiment}

The goal of the present study is to test the hypothesis that some of the same processes are used in mental rotation and motor actions. The stimuli used are images of abstract objects, with no obvious relation to hands or other body parts. If visual image transformation and the production and control of physical movements do in fact share mechanisms, simultaneous mental and motor rotation should interfere with each other. In the present experiment, the participants perform mental rotation in a task like that of Cooper and Shepard (1973) while simultaneously performing a manual rotation (of the handle of a joystick) at an experimentally-controlled, previously-learned angular speed. Our hypothesis leads us to predict interference between the detailed dynamical features of two rotations. Specifically, we expect interference between image and motor rotation that depends on the joint duration, extent, direction and speed of the two rotations. By controlling the motor speed and monitoring the actual motor trajectories, we can probe for detailed interference between the features of the two rotations.

However, we do not predict that every motor action will interfere with mental rotation. Indeed, automatic or passive movements do not require motor planning, and consequently rely differently on the premotor, motor and parietal areas (e.g. see Seitz and Roland, 1992 for motor and premotor areas, and Mountcastle et al. (1975) for parietal cortex). Such movements should elicit little if any visuomotor anticipation, and should therefore interfere less or not at all with mental image transformation. Indeed, pilot studies showed that passive motor rotations had no effect on mental rotation. Nevertheless, it is desirable to have some control over the subject's movement, in order to facilitate the analysis of interference effects. Thus, we have chosen a moderately-difficult motor task in which the participant turns the handle of a joystick at a previously-learned angular speed (within a tolerance range) and in a previously-specified direction.

In typical mental rotation tasks, the participant is shown two figures simultaneously and must decide whether they are identical shapes or mirror images. The subject therefore has two strategies, namely to (mentally) rotate the first image in 
order to bring it into alignment with the second, or vice-versa. Here, we are interested in controlling the directions of both mental and motor rotations in order to examine possible interference. Hence, we must have control over the direction of mental rotation, and thus have used a modified task. In our task, to-be-compared figures are presented sequentially, and an arrow that indicates the orientation of the final figure is presented between the two. The subject, who was encouraged to begin the mental rotation during the intermediate interval between the presentations, was therefore led to mentally rotate the first stimulus to align it with the second, rather than vice versa.

Finally, to eliminate the possibility of spurious interference between the imagery and motor tasks that could arise from simply seeing one's manual rotation, we placed the participants flush against a visual tunnel; at no time did participants see the manual rotation.

\subsection{Method}

\subsubsection{Subjects}

Twelve people volunteered to participate in the experiment (ages 21-29, six men and six women); they were recruited through advertisements placed in universities, and were paid $50 \mathrm{FF} / \mathrm{h}$. None had previous laboratory experience with mental imagery. The subjects were randomly-assigned to one of two groups, 45-90 or 90-45 (see below), with the constraint that each group have three women and three men. In addition, two subjects whose performance on the imagery task was at chance level were disqualified and their data discarded.

\subsubsection{Apparatus}

The experimental apparatus consisted of three parts: a computer screen, a motorcontrolled joystick, and two foot-operated switches. The computer screen was parallel to the subject's frontal plane, at eye level, and about $55 \mathrm{~cm}$ from the subject. (All distances are approximate, given that the subjects had some freedom to adjust chair height and position.) The subject's head was placed flush against a visual tunnel that led to the screen, so that only the screen was seen during the trials.

The center of the motor-controlled joystick was $40 \mathrm{~cm}$ below the computer screen and $55 \mathrm{~cm}$ from the subject. The shaft of the joystick was $15 \mathrm{~cm}$ in length. Its tip could be revolved around a circle $12 \mathrm{~cm}$ in diameter parallel to the subject's frontal plane (i.e. in the plane parallel to the computer screen). The position of the joystick was sampled at a rate of $75 \mathrm{~Hz}$. Movement of the joystick was slightly damped. The joystick was free to rotate about its shaft. The subject's feet rested on two switches.

\subsubsection{Stimuli}

A set of four small, 2D figures were prepared, illustrated in Fig. 1 in their canonical orientations; each figure was created from the rotations and reflections of one basic figure. Each figure was solid white on a black background, subtending a visual angle of about $3^{\circ}$. The resolution of the computer screen was approximately $0.4 \mathrm{~mm} /$ pixel, with the stimuli spanning $90 \times 60$ pixels. A separate set of solid yellow arrows 

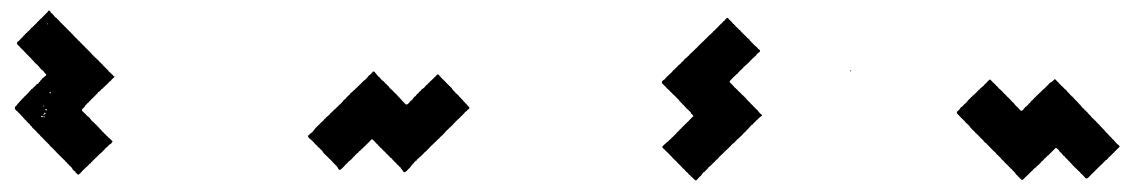

Fig. 1. Stimuli in mental rotation task (canonical orientations).

(length 70 pixels, subtending a visual angle of $3^{\circ}$ ) was prepared, which were pointed in each of the possible orientations associated with the figures.

\subsubsection{Procedure}

On each trial the subject performed two tasks simultaneously, the imagery and the motor tasks. The imagery task was similar to the Cooper and Shepard (1973) 2D mental-rotation task. It consisted of three phases, illustrated in Fig. 2. During Phase 1 (which lasted $5 \mathrm{~s}$ ), the subject viewed a stimulus figure accompanied by an arrow pointing to it from the center of the screen. In Phase 1, the figure was always centered at the top of the screen, in its canonical orientation (i.e. the one shown in Fig. 1); this orientation will be referred to as $0^{\circ}$, with positive angles appearing clockwise. In Phase 2 (which lasted $1.5 \mathrm{~s}$ ) the figure and arrow disappeared, and were replaced by an arrow that pointed from the center of the screen to a different location on the screen, angle $\theta$ from 12 o'clock (in Fig. 2, e.g. $\theta=135^{\circ}$ ). The angle $\theta$ assumed one of eight values: $0^{\circ}, \pm 45^{\circ}, \pm 90^{\circ}, \pm 135^{\circ}$ and $180^{\circ}$ (when $\theta=0^{\circ}$, the arrow in Phase 2 coincided with the arrow in Phase 1). In Phase 3, the arrow from Phase 2 remained on the screen while the figure reappeared at the screen location indicated by the arrow. In addition to being displaced by angle $\theta$ around the screen, the figure was rotated by angle $\theta$ (see Fig. 2 for an example). The figure that reappeared was either a rotation of the original figure in Phase 1, or the rotation of its mirror reflection about a vertical axis.

The subject's task was to decide, as quickly and accurately as possible, whether the figure presented in Phase 3 was identical to the one in Phase 1 (apart from a rotation) or whether it was a mirror image of that figure; if identical, the subject was to press the right foot-switch, and otherwise the left foot-switch. The time between

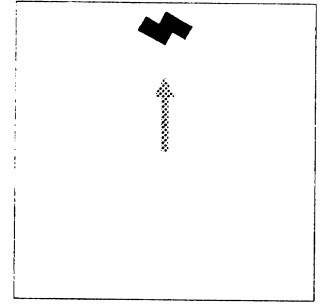

Phase 1

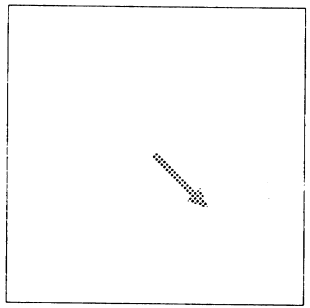

Phase 2

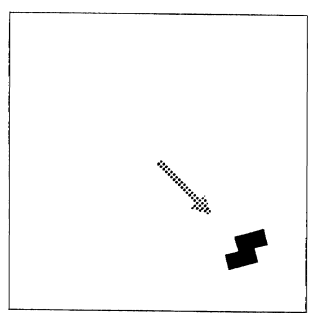

Phase 3

Fig. 2. A typical sequence of stimuli in the mental rotation task (the frame is not displayed, white and black are reversed, gray is yellow). 
the onset of Phase 3 and the subject's response will be referred to as the response time (RT). The subjects' instructions specifically recommended mental rotation as a strategy, and urged them to use the $1.5 \mathrm{~s}$ of Phase 2 to 'begin to imagine what the figure will be like when it reappears at the given orientation'.

The simultaneous motor task required the subjects to revolve the joystick handle in a clockwise or counterclockwise direction at a specified angular speed, $\omega$. (It should be noted that the joystick was revolved, i.e. translated in a circle parallel to the subject's frontal plane; although the joystick was also free to rotate about its shaft, wrist rotation was not part of the task.) The joystick was turned with the right hand; all subjects were right-handed. The direction and speed remained constant for each block of 16 trials. The instructions that appeared on the screen at the beginning of each block explicitly told the subjects to turn clockwise or counterclockwise. The subjects then practiced $360^{\circ}$ turns and received real-time verbal feedback (on the computer screen) on whether their actual, instantaneous angular speed was within a tolerance interval $( \pm 45 \%)$ around the target speed $\omega$, or whether it fell above or below that range. This preliminary motor training continued until the subjects executed two consecutive revolutions without going outside the $45 \%$ tolerance range around $\omega$.

In the trials that made up the subsequent block, the motor task consisted of rotating the joystick in the given direction at an angular speed as close as possible to $\omega$. This motor rotation was synchronized with the imagery task in the following way. The trial always began with the joystick in the upright, $0^{\circ}$ position; the joystick was held fixed during Phase 1 of the imagery task. At the outset of Phase 2 the joystick was released and the subjects were required to begin the motor rotation. The rotation was to be continued, at the constant speed, until a response was given in the imagery task. The motor speed $\omega$ was either $45 \%$ or $90 \%$ s.

The subjects performed the experiment in two sessions; the 45-90 group had motor speed $\omega=45 \%$ d during the first session and $\omega=90 \% \mathrm{~s}$ in the second session, and vice versa for the 90-45 group. Each session consisted of eight blocks of 16 trials each; in addition, a practice block was administered before the beginning of the first session. All trials with errors were repeated in two blocks (one for each direction of motor rotation) following the eight test blocks. There were two kinds of errors: incorrect responses in the imagery task, and trials where the average motor speed fell outside the tolerance range around $\omega$. Within each session, therefore, $\omega$ remained constant, whereas the direction of motor rotation alternated between clockwise and counterclockwise. For each subject, the experiment was a complete factorial design: $2\left(\omega=45,90^{\circ} / \mathrm{s}\right) \times 2$ (counterclockwise or clockwise manual rotation) $\times 4$ (figures in imagery task) $\times 8$ (values of angle $\theta$ in imagery task, in steps of $\left.45^{\circ}\right) \times 2$ (same or reflected). The order of trials was randomized for each subject. At no time during the motor training or during the trials could the subjects see the joystick or their hands, due to the visual tunnel.

\subsection{Results}

Three types of data were collected: the RTs in the imagery task (with a temporal 
resolution of $13 \mathrm{~ms}$ ), the responses in the imagery task (the error rates), and the motor trajectories. Due to computer error, approximately $1.5 \%$ of the imagery data were lost, as well as $5 \%$ of the motor trajectory data. Data were averaged over the
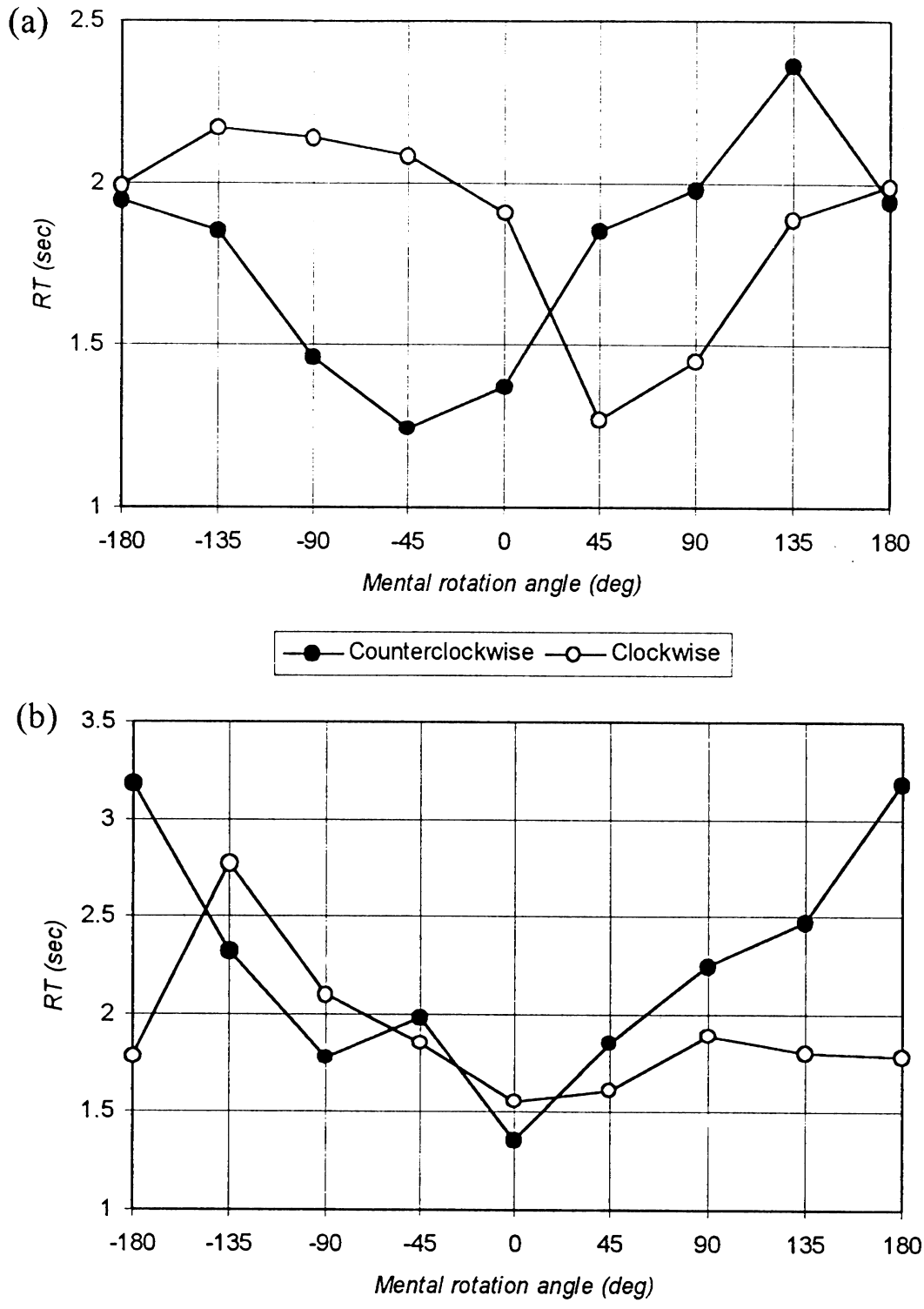

\section{- Counterclockwise -o- Clockwise}

Fig. 3. Response time in the mental rotation task as a function of angle $q$, for different manual rotation conditions (counter-clockwise, clockwise), as well as for the no-motor condition. Data shown is for the first session, for (a) the 45-90 group and (b) the 90-45 group. 
four figures and the two reflection conditions (same/reflected); each data point therefore represents eight trials. For angles other than $\theta=0^{\circ}$ and $180^{\circ}$, trials were characterized as either compatible - where the direction of the mental and motor rotations are the same, or incompatible - where the directions conflict. (Angles $\theta=0^{\circ}$ and $180^{\circ}$ were excluded from analyses that included the compatibility factor.)

The simplest way to exhibit the effect of compatibility is by plotting RT as a function of mental rotation angle $\theta$. This is shown for session 1 in Fig. 3a for the 4590 group, and in Fig. $3 \mathrm{~b}$ for the $90-45$ group. Recall that in our convention, positive $\theta$ means clockwise, so that the right half of each plot represents clockwise mental rotations, and the left half counterclockwise mental rotations. The two curves in each plot represent the two motor conditions: empty circles represent clockwise motor rotations, filled circles counterclockwise motor rotations. The most noticeable effect is the asymmetry between the two motor conditions; clockwise motor rotation facilitates clockwise mental rotation and hinders counterclockwise mental rotation, and vice-versa for counterclockwise motor rotation. Thus, we obtained the predicted compatibility effect. Moreover, perhaps even more strikingly, this effect in the 4590 group (Fig. 3a) is strong enough to displace the minima in the RT curves. In typical mental rotation experiments, without concurrent motor rotation, the minimal RT is at $\theta=0^{\circ}$. In others, it is normally easiest to compare two successive stimuli when they are at the same orientation. This is not so when the mental rotation is accompanied by motor rotation. When subjects perform, say, a slow clockwise motor rotation, they find it easiest to compare the two stimuli when the second stimulus is rotated by $45^{\circ}$ with respect to the first, in the direction of the motor rotation!

An analysis of variance (ANOVA) was performed on the RTs with the following factors: group $(45-90,90-45) \times$ session $(1,2) \times$ direction of motor rotation (clockwise, counterclockwise $) \times$ angle $\left(45^{\circ}, 90^{\circ}, 135^{\circ}\right) \times$ compatibility (compatible, incompatible). First, as predicted, when the mental and motor rotations were compatible (i.e. in the same direction), mental rotation was faster than when the mental and motor rotations were incompatible (but all else was the same), $F(1,10)=7.59$, $P=0.02$. This finding confirms the recent result of Wohlschläger and Wohlschläger (1998). Second, as expected from classic results on mental rotation, RT increases nearly linearly with angle $\theta$ (at roughly $200^{\circ} / \mathrm{s}$ ), $F(2,20)=14.69, P<0.001$. Third, we found that subjects were faster with increased practice; RT decreased by $15 \%$ from session $1(1.91 \mathrm{~s})$ to session $2(1.62 \mathrm{~s}), F(1,10)=5.36, P=0.04$. Finally, whereas the difference between the compatible and incompatible conditions in session 1 was $23.6 \%$ (1.68 and $2.13 \mathrm{~s}$, respectively), the corresponding difference in session 2 was only $3.1 \%$ ( 1.60 and $1.65 \mathrm{~s}), F(1,10)=9.96, P=0.01$ for the interaction of session $\times$ compatibility. An ANOVA on session 1 data alone produced $P=0.01$ for the main effect of compatibility, whereas the same analysis for session 2 alone revealed a statistically-insignificant result, $P=0.42$. The effect of compatibility between the mental and motor rotations is drastically reduced or disappears completely after 200 trials.

There is a detail in Fig. 3 that at first sight may be overlooked, but which is important. As illustrated in Fig. 4, the asymmetry between incompatible and com- 
patible conditions in group 45-90 is largest for $\theta=45^{\circ}$ and decreases as angle $\theta$ becomes larger; the reverse is true for the 90-45 group. This is shown in Fig. 4 as a plot of the difference in RTs between the incompatible and compatible conditions (averaged over positive and negative angles) for the two groups. The fact that the RT differences in Fig. 4 are positive reflects the compatibility effect; here we are calling the reader's attention to the opposite trends as a function of angle $\theta$ for the two groups. This difference is significant: if we fit the two series by straight lines, a sign test yields $P=0.03$ for the difference between the two slopes. This is the first hint of an interaction between the speeds of the motor and mental rotations, as predicted by our hypothesis. If the mental rotation actually follows the motor rotation, the RT asymmetry should be greater for larger motor speeds. The 45-90 group (which rotated at $45 \%$ in the first session) should have larger RT asymmetry when $\theta$ is small, whereas the 90-45 group (which rotated at $90 \%$ s) should have larger RT asymmetry when $\theta$ is large, precisely what appears to be the case (Fig. 4).

We performed another ANOVA on error rates with the same factors as the RT ANOVA. The error rate for a given condition was defined as the number of incorrect (either imagery or motor error) trials for that condition, divided by the total number of trials for that condition in the first eight blocks of each session (not the two at the end that were used to replace trials on which errors had occurred). The results, by session, angle and compatibility, are provided in Table 1. There is an interaction between compatibility and session: in session 1 subjects made $24.5 \%$ more errors on the incompatible trials than on compatible ones, whereas in session 2 the corre-

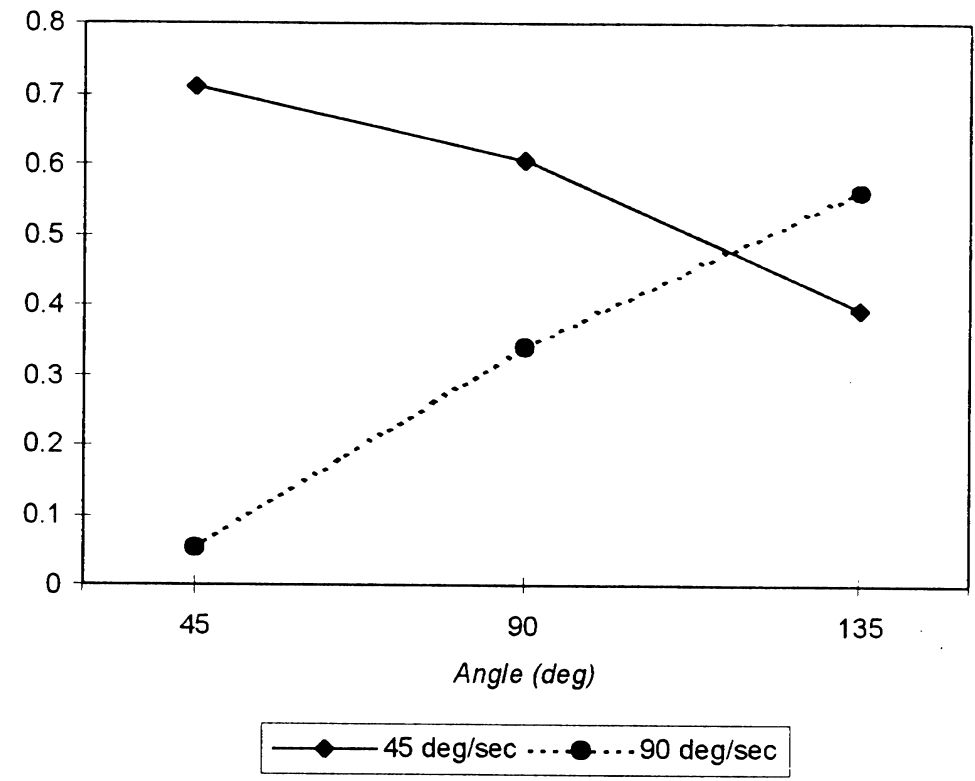

Fig. 4. The difference in response times (RTs) between incompatible and compatible conditions as a function of angle $q$ (averaged over positive and negative angles) for the two groups in session 1 . 
sponding figure is only $1.9 \%(F(1,10)=5.12, P=0.05)$. For session 1 data alone, the effect of compatibility approaches significance with $F(1,10)=4.43, P=0.06$. As found in our analysis of RTs, the effect of compatibility is strong in the first session, and all but disappears in the second session. Not surprisingly, the subjects improved with practice (mean error rate 0.319 in session $1,0.155$ in session 2, $P<0.001)$ and made more errors with larger mental rotation angles $(P=0.01)$. (Neither type of error, motor or imagery, by itself gives a significant compatibility effect; this is most likely due to a loss of statistical power. The trends are, however, to greater errors of both types in the incompatible condition.)

Is there any relation between the speed of mental rotation and the motor speed? Our hypothesis predicts that, all else being equal, higher motor speed, $\omega$, would speed up mental rotation, and lower motor speed would slow it down. This can be checked by comparing the average mental rotation speeds of the two groups of subjects (the 45-90 and the 90-45 groups) in the two sessions. This effect must be distinguished from two uninteresting effects, a possible inter-group difference in mental rotation speed (caused by individual differences among the subjects who happen to be in each group), and an overall speeding-up of mental rotation with practice. Define the mental rotation speed as $|\theta| / \mathrm{RT}$. Averaging by group and session, we obtain the results in Table 2, plotted in Fig. 5.

Both of the uninteresting effects noted above are present in the data: group 90-45 is somewhat slower at mental rotation than group 45-90, which is not surprising given the size of the groups and the large variations in the population; in addition, there is an overall trend for higher speed in the session 2. Superimposed on these, however, is a very large and interesting inter-group difference: group 45-90 (which manually rotated faster in session 2) becomes much faster at mental rotation in session 2, whereas group 90-45 (which manually rotated slower in session 2) actually has somewhat slower mental rotation in session $2, F(1,10)=21.49$, $P<0.001$ for the group $\times$ session interaction. This is strong support for our hypothesis; all else being equal, mental rotation is faster in the presence of fast motor rotation, and slower in the presence of slow motor rotation.

Although we have already seen from the analysis of RTs and errors that there is highly-specific interference between the motor and mental rotation tasks, to reach to the heart of the relationship between mental and motor rotations we must examine the subjects' motor trajectories in more detail. We analyzed the relationship between the angle $\theta$ - the angle between the two stimuli in the imagery task - and what we will call the motor angle, $\theta_{\mathrm{m}}$, the angle through which the subject had rotated the joystick handle at time of response to the imagery task. If mental rotation follows the

Table 1

Error ratio as a function of mental rotation angle, motor/mental rotation compatibility, and session

\begin{tabular}{|c|c|c|c|c|c|c|}
\hline \multirow[b]{2}{*}{ Session } & \multicolumn{2}{|l|}{$q=45^{\circ}$} & \multicolumn{2}{|l|}{$q=90^{\circ}$} & \multicolumn{2}{|l|}{$q=135^{\circ}$} \\
\hline & Compat. & Incomp. & Compat. & Incomp. & Compat. & Incomp. \\
\hline 1 & 0.243 & 0.312 & 0.247 & 0.397 & 0.350 & 0.367 \\
\hline 2 & 0.115 & 0.143 & 0.141 & 0.121 & 0.206 & 0.208 \\
\hline
\end{tabular}




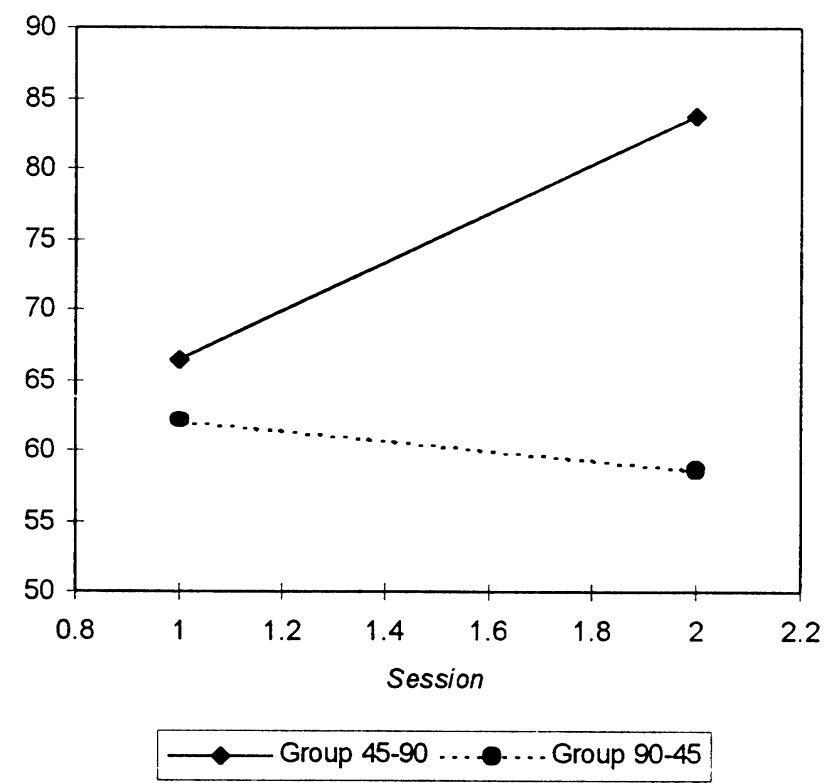

Fig. 5. Mean mental rotation speed by group and experimental session (graph of Table 2). Group 45-90 had slow manual rotation in session 1 and fast manual rotation in session 2, and vice versa for group 90-45.

motor rotation, there should be a positive correlation between the absolute values of the angles $|\theta|$ and $\left|\theta_{\mathrm{m}}\right|$. But this is not a very good test, because even if the two rotations were entirely unrelated we still would expect a positive correlation, given that mental rotation takes more time for larger $|\theta|$ and so does motor rotation for $\left|\theta_{\mathrm{m}}\right|$. If the two rotations in fact proceed together, the correlation between the angles should be much weaker when the rotations are incompatible (i.e. in opposite directions) than when they are compatible: having one's mental image at orientation $\theta_{\mathrm{m}}$ as a result of the motor rotation would not do the subject much good if the test stimulus is at the opposite orientation, $-\theta_{\mathrm{m}}$.

We performed the correlation analysis separately for each subject. All non-error trials with $\theta \neq 0,180^{\circ}$ (in order to allow analysis of the compatibility factor) and $\left|\theta_{\mathrm{m}}\right|<360^{\circ}$ were classified by subject, session and compatibility; in each set the coefficient of correlation between $|\theta|$ and $\left|\theta_{\mathrm{m}}\right|, r$, was calculated. The mean correla-

Table 2

Speed of mental rotation by group and session (in $\%$ )

\begin{tabular}{lll}
\hline Group & Session 1 & Session 2 \\
\hline $45-90$ & 66.4 & 83.8 \\
$90-45$ & 62.1 & 58.6 \\
\hline
\end{tabular}

See also Fig. 5. 
Table 3

Mean correlation coefficients between mental rotation and motor angles

\begin{tabular}{lll}
\hline & Session 1 & Session 2 \\
\hline Compatible & 0.385 & 0.257 \\
Incompatible & 0.083 & 0.264 \\
\hline
\end{tabular}

tions by compatibility and session are summarized in Table 3. Each of the numbers in Table 3 is an average over the individual coefficients of correlation; for example, for session 1 compatibility, $r$ ranged between -0.05 and 0.81 . For the most part, the correlations are positive, and all mean values of $r$, except the low value for session 1 incompatible, are significantly positive at $P<0.01$ (two-tailed $t$-test). In session 1 , the result is as predicted: there is a significant correlation between the angles in the compatible trials, and essentially no correlation $(t(11)=1.07, P=0.3)$ in the incompatible case. Moreover, the relation $r$ (compatible) $>r$ (incompatible) holds individually for 11 out of 12 subjects in session 1 , with an overall $t(11)=2.48, P<0.05$ (two-tailed). As in the results for the RTs and errors, this effect, although robust in the first session, seems to wash out by the second session; giving $t<1$ for the $t$-test for compatibility in the second session. ${ }^{1}$

\section{Discussion}

We found the following effects, all of which support the hypothesis of a tight, dynamical relation between mental and motor rotations. First, motor rotation that is compatible with mental rotation results in faster times and fewer errors in the imagery task than when the two rotations are incompatible. Second, the angle through which subjects rotate their mental images, and the angle through which they rotate the joystick handle are correlated, but only if the directions of the two rotations are compatible. Third, motor rotation modifies the classical inverted Vshaped mental rotation RT function, favoring the direction of the motor rotation, and in some cases even shifts the location of the minimum of this curve in the direction of the motor rotation. Fourth, the preceding effect is sensitive not only to the direction of the motor rotation, but also to the motor speed. A change in the speed of motor rotation can correspondingly slow down or speed up the mental rotation; subjects who manually rotated slower in session 2 also mentally rotated slower, and vice versa.

The interaction between the speeds of the motor and mental rotations persists for

\footnotetext{
${ }^{1}$ One might worry that correlation coefficients do not follow Gaussian statistics, and therefore cannot be directly $t$-tested. This worry is partly allayed by the fact that the relation $r$ (compatible) $>r$ (incompatible) holds individually for 11 out of 12 subjects in session 1, a significant distribution-independent measure. We can also z-transform the $r$ values, as suggested by an anonymous referee, and perform $t$-tests on the new variables. The results are practically identical: all mean values of $r$, except the low value for session 1 incompatible, are still significantly positive at $P<0.01$, and it is still the case that $r$ (compatible) $>r$ (incompatible) at $P<0.05$ in the first session.
} 
both sessions, whereas the interaction between the directions of the two rotations (what we have been calling their compatibility), although robust in session 1 all but disappeared in session 2 . If the mental and motor rotations are initially coupled, their partial decoupling with practice needs to be understood. Consider two explanations of this partial decoupling that are consistent with our hypothesis, one having to do with a reorganization of the mental rotation task, the other with a reorganization of the motor rotation task. First, mental rotation has been found to be extremely sensitive to practice (see, for example, Tarr and Pinker, 1989), and the overall trend to decreasing RTs and errors found in this study is no exception. There is certainly more than one way to compare two images at different orientations, and mental rotation is only one of them (e.g. Hinton and Parsons, 1988). Indeed, a number of subjects spontaneously reported that over the course of the experiment they shifted from mental rotation - a strategy they were instructed to use - to landmark- or memory-based strategies, which, to judge from an overall improvement in RTs, were more efficient. Some of these strategies may involve mental processes that are less coupled - or not coupled at all - to motor mechanisms. For instance, given the small number of stimuli in the experiment, a partial memorization cannot be excluded.

A second possible explanation for the learning effect is that with practice the subjects may have changed their organization of the motor task. Specifically, the task of turning the joystick handle at a fixed speed was at first difficult for most subjects, and required much deliberate planning. By the end of the experiment, however, the task was to a large extent automated, as evidenced by rates of motor error: subjects went outside the tolerance range for the required motor speed on $11.4 \%$ of the trials in the first session, but only on $6.1 \%$ of the trials in the second session $(t(11)=2.12, P=0.03)$. Seitz and Roland (1992) found that the activation of the supplementary motor area (SMA) was modified during the learning and automation of a motor task. SMA activation was highest at the beginning of the learning phase, and decreased as the performance of the task became automatic. According to our hypothesis, it is precisely the motor planning and anticipation areas such as the SMA that should be responsible for the transformation of mental images. Automation of the motor task in our experiment could therefore account for the partial decrease of the motor-imagery interaction in the second session. The practice effect that we have observed thus provides indirect evidence that it is the higher motor areas, such as the SMA and premotor cortex (whose activation decreases with practice) that are responsible for the observed interaction between action and imagery. We thus have two possible explanations for the learning effect, both of which are consistent with our hypothesis. These explanations are not mutually contradictory, and may both contribute to the learning effect.

In another PET study, Deiber et al. (1991) showed that premotor cortex and the SMA are more strongly activated in endogenous movement where the subject, for example, has freedom to choose the direction of a movement on each trial, than when the direction of the movement is pre-learned or imposed in some other way. It would be instructive, therefore, to manipulate the involvement of motor planning mechanisms by repeating the present study with a condition in which the subject 
randomly chooses the direction of rotation on each trial. In light of the results of Deiber et al., the motor-imagery interaction should be even stronger in such a procedure than that seen in the present study.

One could try to argue that our results do not really support our hypothesis, in the following way. On each trial, the subject had to remember the direction of the motor rotation, and this information could have been stored in the form of a visual image (even though the subjects could not see their hands). The fact that mental rotations were faster and less error-prone when the motor rotation was in the same direction could then be easily explained as a result of a purely visual conflict between the two opposing directions; our results would then simply be evidence for fluent intermodal transfer between the motor and visual imagery systems, rather than for common mechanisms.

In fact, if our sole result were the compatibility effect, an interpretation in terms of our imagery-motor hypothesis would be suspect. Indeed, the results of Wohlschläger and Wohlschläger (1998), with nothing else to back them up, would be open to the above criticism. The critique can be countered given the present findings, however. We found very specific, positive correlations not only between the directions but also between the speeds, as well as the angles. These correlations cannot be explained by a simple interference between two forms of spatial memory.

We have shown a specific, detailed interaction between the rotation of a visual mental image and motor rotation of a joystick handle. This interaction is much more than a dual-task effect; it depends crucially on the details of the two rotations: their directions, their final positions, and their speeds. The interaction is all the more surprising, because it appears without any visual feedback to motor rotation; the subject can see neither the joystick nor his or her hands during the trials, and the motor rotation has strictly no effect on the visual stimuli or on anything else the subject sees.

Although we cannot propose a detailed model, visuomotor anticipation - the prediction of the visual consequences of an about-to-be-executed motor action is likely to be the mechanism that drives at least some types of mental-image transformation, and in particular mental rotation. A good candidate for the locus of the interaction would therefore be the motor planning and anticipation systems, most likely located in premotor and posterior parietal cortex.

In short, the present results provide strong evidence that motor processes are used even when abstract objects are mentally rotated. These findings are as predicted if mental rotation is produced, at least partly, in conjunction with the motor system. 1986

\section{Acknowledgements}

M.W. wishes to thank the LPPA for its hospitality, and especially Joseph A. McIntyre and Sylvain Hanneton for help with equipment and Gilles Leone and Michelange Amorim for carefully reading the manuscript. 


\section{References}

Andersen, R.A., Snyder, L.H., Bradley, D.C., Xing, J., 1997. Multimodal representation of space in the posterior parietal cortex and its use in planning movements. Annual Review of Neuroscience 20, 303 320.

Berthoz, A., 1997. Le Sens du Mouvement. Odile Jacob, Paris.

Cohen, M.S., Kosslyn, S.M., Breiter, H.C., DiGirolamo, W.L., Thompson, W.L., Anderson, A.K., Bookheimer, S.Y., Rosen, B.R., Belliveau, J.W., 1996. Changes in cortical activity during mental rotation. A mapping study using functional MRI. Brain 119, 89-100.

Cooper, L.A., Shepard, R.N., 1973. Chronometric studies of the rotation of mental images. In: Chase, W.G. (Ed.), Visual Information Processing. Academic Press, New York.

Cooper, L.A., Shepard, R.N., 1975. Mental transformations in the identification of left and right hands. Journal of Experimental Psychology: Human Perception and Performance 1, 48-56.Deiber, M.P., Passingham, R.E., Colebatch, J.G., Friston, K.J., Nixon, P.D., Frackoviak, R.R.J., 1991. Cortical areas and the selection of movement: a study with positron emission tomography. Experimental Brain Research 84, 393-402.

Duhamel, J.-R., Colby, C.L., Goldberg, M.E., 1992. The updating of the representation of visual space in parietal cortex by intended eye movements. Science 255, 90-92.

Georgopoulos, A.P., Lurito, J.T., Petrides, M., Schwartz, A.B., Massey, J.T., 1989. Mental rotation of the neuronal population vector. Science 243, 234-236.

Georgopoulos, A.P., Massey, J.T., 1987. Cognitive spatial-motor processes. 1. The making of movements at various angles from a stimulus direction. Experimental Brain Research 65, 361-370.

Held, R., Hein, A. Adaptation of disarranged hand-eye coordination contingent upon re-afferent stimulation. Perceptual Motor Skills 8, 87-90.

Hietanen, J.K., Perrett, D.I., 1996. Motion sensitive cells in the macaque superior temporal polysensory area: response discrimination between self-generated and externally generated pattern motion. Behavioural Brain Research 76, 155-167.

Hinton, G.E., Parsons, L.M., 1988. Scene-based and viewer-centered representations for comparing shapes. Cognition 30, 1-35.

Jeannerod, M., 1997. The cognitive neuroscience of action. Blackwell, Oxford, UK.

Kirsh, D., Maglio, P., 1994. On distinguishing epistemic from pragmatic action. Cognitive Science 18, 513-549.

Kosslyn, S.M., 1980. Image and mind. Harvard University Press, Cambridge, MA.

Kosslyn, S.M., 1994. Image and brain. MIT Press, Cambridge, MA.

Kosslyn, S.M., DiGirolamo, G.J., Thompson, W.L., Alpert, N.M., 1998. Mental rotation of objects versus hands: neural mechanisms revealed by positron emission tomography. Psychophysiology, in press.

Mountcastle, V.B., Lynch, J.C., Georgopoulos, A., Sakata, H., Acuna, C., 1975. Posterior parietal association cortex of the monkey: command functions for operations within extrapersonal space. Journal of Neurophysiology 38, 871-908.

Parsons, L.M., 1987. Imagined spatial transformations of one's hands and feet. Cognitive Psychology 19, $178-241$

Parsons, L.M., 1994. Temporal and kinematic properties of motor behavior reflected in mentally simulated action. Journal of Experimental Psychology: Human Perception and Performance 20, 709-730.

Parsons, L.M., Fox, P.T., Downs, J.H., Glass, T., Hirsch, T.B., Martin, C.C., Jerabek, P.A., Lancaster, J.L., 1995. Use of implicit motor imagery for visual shape discrimination as revealed by PET. Nature 375, $54-58$.

Pellizzer, G., Georgopoulos, A.P., 1993. Common processing constraints for visuomotor and visual mental rotations. Experimental Brain Research 93, 165-172.

Rieser, J.J., Guth, D.A., Hill, E.W., 1986. Sensitivity to perspective structure while walking without vision. Perception 15, 173-188.

Seitz, R.J., Roland, P.E., 1992. Learning of sequential finger movements in man: a combined kinematic and positron emission tomography (PET) study. European Journal of Neuroscience 4, 154-165. 
Sekiyama, K., 1982. The kinesthetic aspects of mental representation in the identification of left and right hands. Perception and Psychophysics 32, 89-95.

Shepard, R.N., Cooper, L.A., 1982. Mental images and their transformations. MIT Press/Bradford Books, Cambridge, MA.

Shepard, R.N., Judd, S.A., 1976. Perceptual illusion of rotation of three-dimensional objects. Science 191, 952-954.

Shepard, R.N., Metzler, J., 1971. Mental rotation of three-dimensional objects. Science 171, 701-703.

Sirigu, A., Duhamel, J.-R., Cohen, L., Pillon, B., Dubois, B., Agid, Y., 1996. The mental representation of hand movements after parietal cortex damage. Science 273, 1564-1568.

Tarr, M.J., Pinker, S., 1989. Mental rotation and orientation-dependence in shape recognition. Cognitive Psychology 21, 233-282.

Wohlschläger, A., Wohlschläger, A., 1988. Mental and manual rotation. Journal of Experimental Psychology: Human Perception and Performance 24, 397-412. 